\title{
Modification of the Method of Determination of Microbicide Activity of Neutrophils of Blood of Animals
}

\author{
Korablyeva Tatyana Rafailovna ${ }^{1 *}$ and Senchuk Ivan Viktorovich ${ }^{2}$ \\ ${ }^{1}$ Department of Microbiology, VI Vernadsky Crimean Federal University, Russia \\ ${ }^{2}$ Academy of Life and Environmental Sciences FSAEI HE, VI Vernadsky Crimean Federal University, Russia \\ *Corresponding author: Korablyeva Tatyana Rafailovna, Doctor of Veterinary Science, Head of the Department of Microbiology, VI Vernadsky Crimean \\ Federal University, Russia, Email: astemenkolp@gmail.com
}

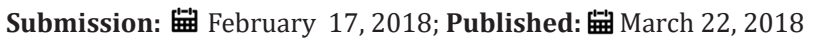

\begin{abstract}
The article contains information of the modification of the method for determining the microbicidal activity of neutrophils in animal's blood, which makes it possible to accelerate the evaluation of the obtained results and to increase the accuracy of the studies performed by using the nitrous tetrazole as a detection detector in to the diformazine graphical editor.

Keywords: Cellular immunity; Microbicidal activity; Neutrophils; Nitroblue tetrazole
\end{abstract}

\section{Introduction}

One of the main methods of studying of the functional activity of neutrophilic granulocytes of blood is a test with nitroblue tetrazolium (NBT). It is based on the ability of colorless NBT to be reduced by oxygen radicals in dark blue dizaphase. As the prototype of the technique, we selected the most informative method of studying the oxygen-dependent metabolism of peripheral blood neutrophils using the laboratory strain Staphylococcus aureus strain P-209 as a test system. The results of the reaction are recorded visually or photometrically. However, the visual account of the NBT- test is subjective and does not allow for a quantitative assessment of the activity of neutrophilic granulocytes. At the same time, most photometric versions of the NBT- test use toxic solvents and significant volumes of reagents [1]. Oxygen-dependent germicidal activity of neutrophils in blood was determined by NBT test (+NBT\%), based on the reduction of the absorbed soluble dye of nitroblue tetrazolium into the insoluble diphormazane. Methods for determining the absorptive activity of neutrophils in peripheral blood, using as test system colored particles of latex with a diameter of $3 \mu \mathrm{m}$ [2], studies of bacterial phagocytosis by neutrophils of capillary blood [3] are also described in literature. There is also a method for studying the metabolic activity of neutrophils [4]. Any work describing the rapid methods of determining the metabolic activity (reserve index) of peripheral blood neutrophils using nitrous tetrazolium as a regain detector in the diformazan of graphic tools of a computer image editor program has not been found in the scientific literature.
Materials and Methods

\section{Reagents}

1. $0.1 \%$ aqueous solution of nitrosinium tetrazolium (pph, $\mathrm{hp}, \mathrm{ppm}$ ): $0.1 \mathrm{~g}$ of nitroblue tetrazolium (NBT) is added to a $100 \mathrm{ml}$ volumetric flask and brought to the mark with distilled water. The examined blood was mixed with an equal volume of $0.1 \%$ solution of NBT (company «Sigma" USA) prepared on the phosphate buffer ( $\mathrm{pH}=7.2)$.

2. $0.9 \%$ solution of sodium chloride (physiological solution). The solution must be sterile.

3. Suspension of the inactivated agar culture of Staphylococcus aureus strain 209-P in physiological saline.

\section{Equipment}

Thermostat, test tubes, serological plates, pipettes, scales, personal computer, digital camera. The course of the definition for the stimulated NBT-test, heparin-stabilized blood is used. It must be dispensed by $0.1 \mathrm{ml}$ in wells of serological plates. Then mix each sample with $0.1 \mathrm{ml}$ of slurry of a killed daily agar culture of Staphylococcus aureus strain 209-P (test system) prepared in sterile saline and add $0.1 \%$ aqueous solution of nitroblue tetrazolium (NBT). Investigation of the absorption phase of phagocytosis and the metabolic activity of peripheral blood neutrophils was performed in patients of calves aged 10 days of red Ukrainian breed 
$(n=10)$. To set the un-stimulated NBT- test, heparin-stabilized blood is poured into $0.1 \mathrm{ml}$ of wells into the wells of serological plates; each sample is mixed with $0.1 \mathrm{ml}$ of sterile saline and $0.1 \%$ aqueous solution of nitroblue tetrazolium. To ensure the identical reaction conditions for the evaluation of the stimulated and unstimulated NBT-test, one microbiological plate should be used. In this case, it is recommended to adhere the following arrangement: the upper row of the wells of the plate is the control; the second row from the top is the un-stimulated (spont.) NBT test, the bottom row of the wells of the plate is the stimulated NBT-test (stim.). Then incubate in a thermostat for 30 minutes at $37 \pm 0.1^{\circ} \mathrm{C}$. After incubation, the wells of the serological plates with the blood being examined are photographed; the image is transferred as a file (in jpg format) to a personal computer. We propose to evaluate the results of the study using the image editor program «PicPick». The choice of this graphic editor is due to the possibility of its free download, the ability to install on a computer with a small performance with the most common Microsoft Windows 10, 8.1, 8, 7, Vista, XP. In the computer program "PicPick" with the option "New task" they open a photo file with the recorded results of the NBT- test. Using the computer mouse, place the program cursor (the cursor is in the "Graphic Tools" option) in the center of the image of the well with the blood being examined and conduct the detection of color parameters using the built-in scale of the color palette. Set the digital indicators that reflect the color gamma of red color (the color palette parameter is indicated by the Latin letter $\mathrm{R}$ ) and blue (the color palette parameter is indicated by the Latin letter B). Determine the ratio of the parameters of red and blue in the contents of the wells of the blood of each animal individually in the stimulated (NBT stim.) and the un-stimulated version of the NBT test (NBT spont.). The stimulation index (IS) of the NBT is calculated as the ratio of the established indices according to the formula:

\section{IS = NBT stim. / NBT spont.}

The technical result of our modification of this technique is to reduce the time, reduce the cost of research without reducing the sensitivity of the proposed test, which allows optimizing the research by using nitro-blue tetrazolium as a detection detector in the diformazan of graphic tools of the «PicPick» computer image editor software. The technique implies the setting of a stimulated and un-stimulated NBT- test, while the reserve index of the microbicidal activity of blood neutrophils is estimated by the definition of the stimulation index of the NBT-test, the ratio of $\%$ formazan positive neutrophils in the stimulated NBT-test to \% formazan positive neutrophils in the un-stimulated NBT- test.

To determine the comparability of the results obtained, the conventional cytological method for determining the parameters of the NBT test was used in parallel [1], in which the estimation of the reserve of microbicidal activity of blood neutrophils is carried out by determining the stimulation index of the NBT-test) is the ratio of $\% \mathrm{PC}$ in the stimulated NBT-test to \%PC in the un-stimulated NBTtest (where \% $\mathrm{PC}$ is the percentage of formalin-positive cells). The results of the evaluation of the NBT-test of blood neutrophils in calves are presented in the Table 1 . The obtained digital data were processed by the method of variation statistics. From the data given in the Table 1 , it can be seen that there is no statistically significant difference between the indices of the stimulation index of the NBTtest obtained by the results of the conventional method and the modified technique. This allows us to affirm the comparability of the data obtained.

Table 1: Indicators of the results of the evaluation of the NBT-test of blood neutrophils in calves.

\begin{tabular}{|c|c|c|c|c|c|}
\hline \multicolumn{2}{|c|}{$\begin{array}{c}\text { Indicators of the Standard Cytological Method for the Evaluation of } \\
\text { the Results of the NBT-Test }\end{array}$} & \multicolumn{2}{|c|}{$\begin{array}{c}\text { Indicators for the Evaluation of the Results of the NBT-Test Using the } \\
\text { Computer Program Steam }\end{array}$} \\
\hline Stim. NBT-Test & NBT Test \% Spont. & IS NBT & Stim. NBT-Test & Spont. NBT-Test & IS NBT \\
\hline $9.22 \pm 2.2$ & $37.8 \pm 2.9$ & $0.24 \pm 0,05$ & $0.47 \pm 0.06$ & $1.66 \pm 0.23$ & $0.28 \pm 0.01$ \\
\hline
\end{tabular}

\section{Conclusion}

The novelty of the invention consists in using an accessible computer image editing program to detect the color change of the blood under examination when the NBT-test is set up during the reduction of nitroblue tetrazolium to blue dibazan. This allows rapid diagnosis of changes in the microbicidal reserve of blood neutrophils in animals makes it accessible and does not reduce its informative value. The method makes it possible to significantly save research time, does not require additional reagents for coloring blood smears for the purpose of visualization of all blood cells. The technical solution is of great economic importance. It saves time and reagents, does not require expensive equipment, and creates a previously unknown possibility of express diagnostics of cell disorders in the nonspecific reactivity of animals, which can be used as a basis for diagnosing various pathological conditions.

\section{Acknowledgment}

The authors are grateful to Academy of bioresurse and environmental sciences for provision of logistics, and the university staffs for material and logistic supports, and their cooperation to bring this research in to completion.

\section{References}

1. Park BH, Fikrig SM, Smithwich EM (1968) Infection and nitroblue-tetrazolium reduction by neutrophils. A diagnostic acid. Lancet 2(7567): 532-534. 
2. Medvedev N, Chalenko VV (1991) A method of studying the absorbent phase of phagocytosis. Lab Delo 2: 19-20.

3. Nesterova, Slynko LI, Svetlichnaya MA (1989) Methodological recommendations. Krasnodar, Russia.
4. Vicksman ME, Mayanskii AN (1980) Characteristic of the opsonin factors according to the reaction of nitroblue tetrazolium reduction by human neutrophils Bulletin of Experimental Biology and Medicine 89(2): 214215.

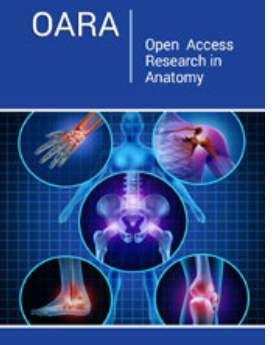

\section{Open Access Research in Anatomy}

\section{Benefits of Publishing with us}

- High-level peer review and editorial services

- Freely accessible online immediately upon publication

- Authors retain the copyright to their work

- Licensing it under a Creative Commons license

- Visibility through different online platforms 